\title{
Association of Anthropometric Measurement With Ultrasound- Diagnosed Non Alcoholic Fatty Liver Disease In Dhaka Medical College Hospital
}

\author{
Farhana Sultana ${ }^{1}$, Mohammad Shamsuddoha Sarker Shanchay ${ }^{2}$, Faruque Ahmed ${ }^{3}$
}

\begin{abstract}
Introduction: Nonalcoholic fatty liver disease (NAFLD) is an increasingly recognized cause of liver disease worldwide. The present study was conducted with the aim to determine the association between anthropometric measurement and incidence of NAFLD among the adult population.

Material and Methods: A hospital based cross sectional study was performed among 240 patients attending outpatient department of Hepatology, Gastroenterology and admitted in patient of Medicine, Hepatology, Gastroenterology department of Dhaka Medical College Hospital, who are Ultrasound diagnosed NAFLD, fulfilled the inclusion criteria. The Study was done from August, 2013 to February, 2014 in Dhaka Medical College Hospital, Dhaka, Bangladesh. Then a pro-forma was made and filled it by direct interview, reading the remarks of the consultant about the patient written on the file, using the hospital lab results and by discussing the patient's file remarks with the medical officer on duty. NAFLD was confirmed by Ultrasonography. Relevant Laboratory investigations were done to assess severity and prognosis. All subjects underwent anthropometric measurement which included height, weight, waist circumference, waist hip ratio (WHR), waist height ratio (WHtR). Body mass index (BMI) and were calculated using the standard formula.

Results: Out of 240 patients, 5 I (2I.25\%) were found to be diagnosed with NAFLD (95\%Cl=22.8-27.8) subjects. The cases of NAFLD were significantly higher in males (55.0\%) as compared to females (45\%) $(R R=1.17,95 \% C l=1.14-2.58, p=0.008)$. The $B M I$, waist circumference, $W H R$ and $W H t R$ were significantly $(p<0.000 I)$ associated with NAFLD. Likewise, NAFLD was found significantly $(p<0.0001)$ higher among obese and overweight subjects. However, in multivariate logistic regression analysis, sex (adjusted $O R=I 1.30$, $95 \% \mathrm{Cl}=3.44-37.1 \mathrm{I}, \mathrm{p}<0.000 \mathrm{I}$ ), waist circumference (adjusted $\mathrm{OR}=1.27,95 \% \mathrm{Cl}=1.56-1.39, p<0.000 \mathrm{I}$ ), hip circumference (adjusted $O R=0.90,95 \% C l=0.82-0.99, p=0.04$ ) and $B M I$ (adjusted $O R=I .83,95 \% C l=I .10$ 2.20, $p=0.001$ ) were significantly associated with NAFLD.
\end{abstract}

Conclusion: It can be concluded that higher anthropometric measurements were significantly associated with NAFLD.

Keywords: NAFLD, Anthropometric measurement, BMI, waist circumference, WHR, WHtR

(c) (1) (5) (2)

(c) Attribution-NonCommercial-NoDerivatives 4.0 International License, which permits use, distribution and reproduction in any medium, provided the original work is properly cited, is not changed in any way and it is not used for commercial purposes.

Received: I 8 November, 2020;
Accepted: 02 December, 2020
1. Registrar, Medicine, Dhaka Medical College \& Hospital.

2. Resident Physician, Medicine, 300 bedded Hospital, Narayangonj

3. Professor and Head, Dept. of Gastroenterology, Dhaka Medical College \& Hospital.

Corresponding Author: Dr. Farhana Sultana, Registrar, Medicine, Dhaka Medical College Hospital, Phone: 01911250094 e-mail: dr.rumi36@gmail.com

\section{Introduction:}

Non-alcoholic fatty liver disease (NAFLD) is the term used to describe the alcohol-like liver injury that occurs in the absence of alcohol abuse (alcohol consumption of over 20 $\mathrm{g}$ /day excludes the condition) ${ }^{1}$. It represents a spectrum of liver diseases characterized mainly by macrovesicular steatosis that occurs in the absence of alcohol consumption in amounts considered injurious to the liver ${ }^{2}$. The hepatic 
histology can vary from isolated hepatic steatosis alone to steatohepatitis and are referred to as non-alcoholic fatty liver (NAFLD) and non-alcoholic steatohepatitis (NASH) respectively. ${ }^{3}$ The incidence of NAFLD is increasing, become a common chronic liver disease and a critical problem threatening the human health. NAFLD affects 10 to $24 \%$ of general population in various countries. The prevalence increases to $57.5 \%{ }^{4}$ to $74 \%{ }^{5}$ in obese persons. NAFLD affects $2.6 \%$ of children and $22.5 \%$ to $52.8 \%$ of obese children $^{6}$. The prevalence of type 2 Diabetes Mellitus varied between 10 to $75 \%$ and the prevalence of hyperlipidemia varied between 20 to $92 \%{ }^{7}$. Primary causes of non-alcoholic fatty liver disease is metabolic syndrome and Secondary cause are nutritional (protein-calorie malnutrition, rapid weight loss, gastrointestinal bypass surgery, total parental nutrition, etc.) drugs (glucocorticoids, estrogens, tamoxifen, amiodarone, methotrexate, diltiazem, valproate, aspirin, etc.) metabolic (lipodystrophy, hypopituitarism, hypothyroidism) toxins, infections and others (eg. Rheumatoid arthritis and systemic lupus erythematosus $)^{8}$. Ultrasound of HBS is comparatively cheap and readily available but is less sensitive at detecting minimal $(<30 \%)$ steatosis or among obese patients (BMI of 35-40 $\mathrm{kg} / \mathrm{m} 2)^{9}$ Thus a negative ultrasound does not necessarily exclude NAFLD. Although it is sensitive (85-95\%), but nonspecific (positive predictive value $62 \%$ ) $^{2}$. Liver biopsy is the gold standard for diagnosis of NAFLD, but the large number of afflicted subjects, invasive nature of a liver biopsy and the lack of effective treatment, there is often a reluctance to pursue this diagnosis with vigor and many patients are left with a diagnosis of suspected NAFLD. The need to perform a biopsy and make these distinctions continue to generate controversy. The answer to this question is linked to the critical final question: even if the diagnosis is made, is effective treatment available? ${ }^{2}$

In the present study, we emphasize to find out the easy screening tool for prediction of NAFLD. Patients who are ultrasound diagnosed NAFLD, will be recruited, and the four anthropometric indicators including waist circumference (WC), waist-to-hip ratio (WHR), waist-to-height ratio (WHtR), and body mass index (BMI) will be determined.

It can produce awareness among all level of medical practitioners, minimizing diagnostic hazards and thereby reducing both mortality and morbidity among the population of this country as well as the world and also there is a message for the health policy makers about importance of timely diagnostic and therapeutic interventions. More studies in this field are needed to prevent this emerging disease.

\section{Material and Methods:}

A hospital based cross sectional study was performed among 240 patients attending outpatient department of Hepatology, Gastroenterology and admitted in patient of the Medicine, Hepatology, Gastroenterology department of Dhaka Medical College Hospital, who are Ultrasound diagnosed NAFLD, fulfilled the inclusion criteria. The Study was done from August, 2013 to February, 2014 in Dhaka Medical College Hospital, Dhaka, Bangladesh. Then a pro-forma was made and filled it by direct interview, reading the remarks of the consultant about the patient written on the file, using the hospital lab results and by discussing the patient's file remarks with the medical officer on duty. NAFLD was confirmed by Ultrasonography. Relevant Laboratory investigations were done to assess severity and prognosis. . All subjects underwent anthropometric measurement which included height, weight, waist circumference, waist hip ratio (WHR), waist height ratio (WHtR). Body mass index (BMI) and were calculated using the standard formula.

\section{Results}

A total of 240 subjects were studied. Among them 51 (21.25\%) are USG diagnosed case of NAFLD and 189 are Non NAFLD and out of 51 NAFLD cases $28(54.90 \%)$ are male and $23(45.10 \%)$ are female.

Table 3.1

Prevalence of NAFLD

\begin{tabular}{ll}
\hline Group & No of patient (\%) \\
\hline NAFLD & $51(21.25 \%)$ \\
Non NAFLD & $189(78.75 \%)$ \\
\hline
\end{tabular}

Table 3.1 shows that prevalence of NAFLD subjects which is about $21.25 \%$ in our society. It is about one fourth of the samle population in comparison with Non NAFLD subjects.

Table 3.2

Distribution of sex among NAFLD subjects

\begin{tabular}{llc}
\hline Sex & NAFLD & Non NAFLD \\
\hline Male & $28(54.90 \%)$ & $77(40.74 \%)$ \\
Female & $23(45.10 \%)$ & $112(59.26 \%)$ \\
\hline
\end{tabular}

Table 3.2: shows that the cases of NAFLD is slightly higher in males $(54.90 \%)$ as compared to females $(45.10 \%)$ $(\mathrm{RR}=1.17,95 \% \mathrm{CI}=1.14-2.58, \mathrm{p}=0.008)$. 
Table 3.3

Distribution of age among NAFLD subjects

\begin{tabular}{lcc}
\hline Age & NAFLD & Non NAFLD \\
\hline Mean & $36.30 \pm 10$ & $37.30 \pm 10.17$ \\
$25-34$ & $3(5.88 \%)$ & $15(7.94 \%)$ \\
$35-44$ & $20(39.22 \%)$ & $75(39.68 \%)$ \\
$45-54$ & $14(27.45 \%)$ & $65(34.39 \%)$ \\
$55-64$ & $12(23.53 \%)$ & $24(12.70 \%)$ \\
$>65$ & $2(3.92 \%)$ & $10(5.29 \%)$ \\
\hline
\end{tabular}

$(\mathrm{P}=0.001)$

Table 3.3 showing that mean age of study population is $36.30 \pm 10$ and highest incidence of NAFLD is in age group of $35-44(39.22 \%)$ and then $45-54(27.45 \%)$ age group. The least incidence is in age group of $>65(3.92 \%)$.

Table 3.4

Comparison of BMI among NAFLD and Non NAFLD subjects

\begin{tabular}{lcc}
\hline BMI & NAFLD & Non NAFLD \\
\hline Underweight $(<18.50)$ & $4(7.84 \%)$ & $21(11.11 \%)$ \\
Normal $(18.50-24.99)$ & $3(5.88 \%)$ & $96(50.79 \%)$ \\
Overweight $(\geq 25.00)$ & $26(50.99 \%)$ & $56(29.63 \%)$ \\
Obese $(\geq 30.00)$ & $18(35.29 \%)$ & $16(8.47 \%)$ \\
\hline
\end{tabular}

$\mathrm{p}<0.0001$

Table 3.4: showing that incidence of NAFLD is higher in increased BMI Group especially in overweight which is about $50.99 \%$ and then in obese patient which is about $35.29 \%$.It reveals that BMI has strong association with Non alcoholic fatty liver disease which may used as a screening tool of NAFLD.

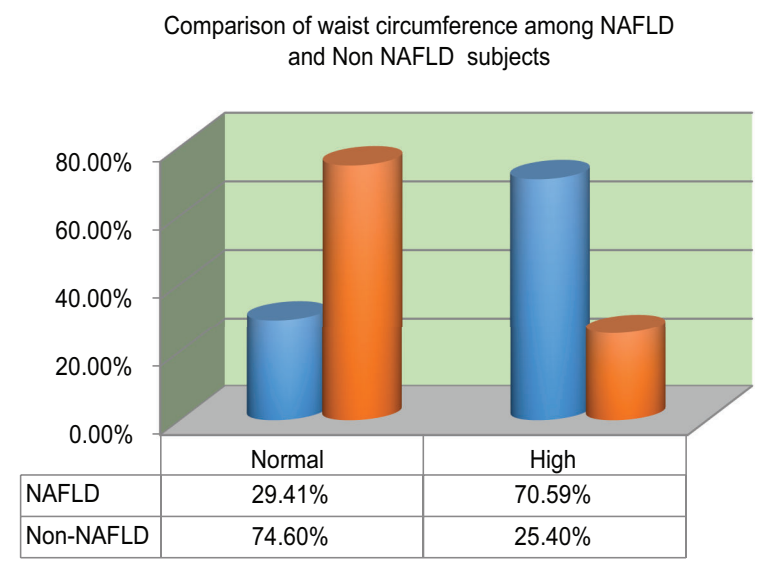

Figure 3.1: Comparison of waist circumference (WC) among NAFLD and Non NAFLD subjects
Figure 3.1: shows that Non alcoholic fatty liver disease has strong association with increased waist circumference, which is about $70.59 \%$ and should be used as an easy screening tool for diagnosis of NAFLD in a low resource country.

Table 3.5

Comparison of waist hip ratio (WHR) among NAFLD and Non NAFLD Subjects

\begin{tabular}{lcc}
\hline waist hip ratio (WHR) & NAFLD & Non NAFLD \\
\hline Normal & $14(27.45 \%)$ & $142(75.13 \%)$ \\
Hig $h(>0.9$ for men and & $37(72.55 \%)$ & $47(24.87 \%)$ \\
$>0.85$ for women & & \\
\hline
\end{tabular}

$\mathrm{p}<0.0001$

Table 3.5: Shows that waist hip ratio (WHR) has strong correlation with NAFLD. It is about $72.55 \%$ and in comparison with Non NAFLD Group which is about $24.87 \%$, is very high.

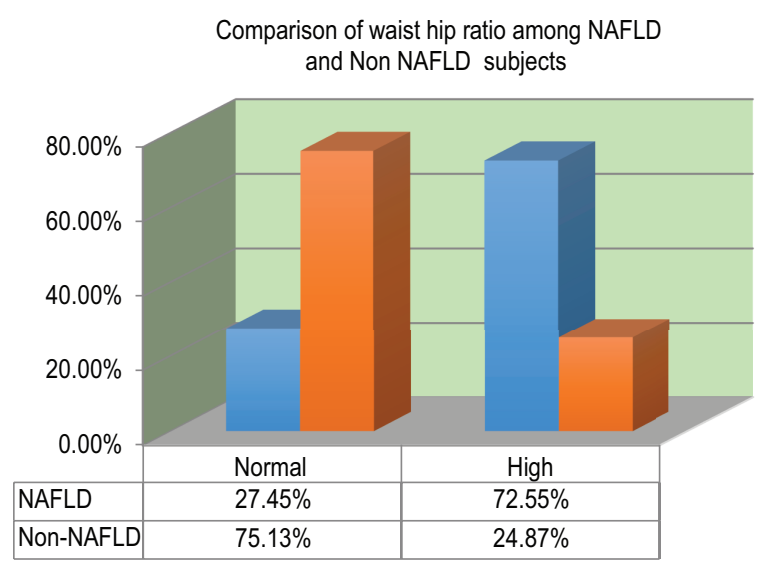

Figure 3.2: Comparison of waist hip ratio (WHR) among NAFLD and Non NAFLD Subjects

Figure 3.2 : shows that waist hip ratio (WHR) has strong correlation with NAFLD. It is about $72.55 \%$ and in comparison with Non NAFLD Group which is about $24.87 \%$, is very high.

Table 3.7

Comparison of waist height ratio (WHtR) among NAFLD and Non NAFLD subjects

\begin{tabular}{lcc}
\hline Waist height ratio (WHtR) & NAFLD & Non NAFLD \\
\hline Normal & $16(31.37 \%)$ & $140(74.07 \%)$ \\
High $(>0.6)$ & $37(68.63 \%)$ & $49(25.93 \%)$ \\
\hline $\mathrm{p}<0.0001$ & &
\end{tabular}

Table 3.7: shows that waist height ratio (WHtR) is significantly higher in NAFLD patient than Non NAFLD patient. 


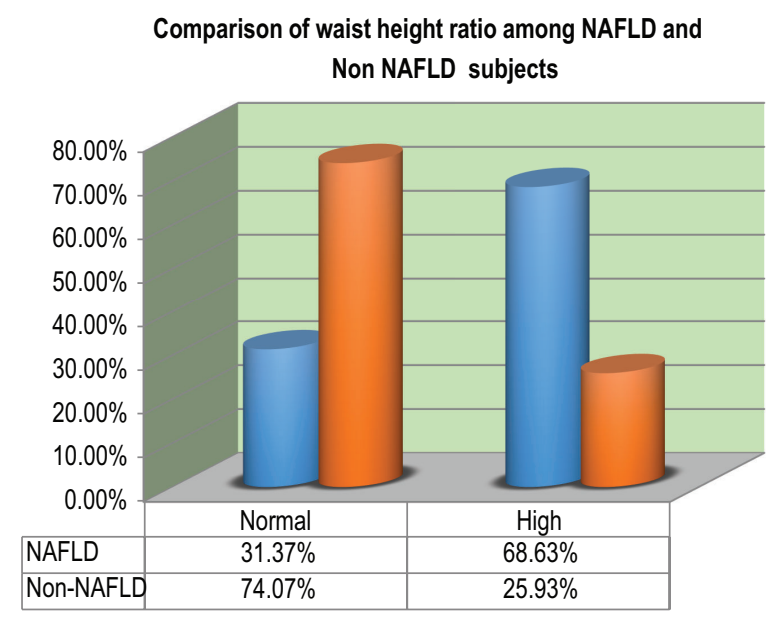

Figure 3.3: Comparison of waist height ratio (WHtR) among NAFLD and Non NAFLD subjects

Table 3.7

NAFLD with other co-morbid condition

\begin{tabular}{lcc}
\hline Metabolic syndrome & NAFLD & Non NAFLD \\
\hline DM & 24 & 30 \\
Hypertension & 20 & 37 \\
Dyslipidaemia & 37 & 53 \\
\hline
\end{tabular}

Table 3.7 showing incidence of NAFLD with DM is high, and there is correlation of NAFLD with dyslipidaemia.
NAFLD with metabolic syndrome

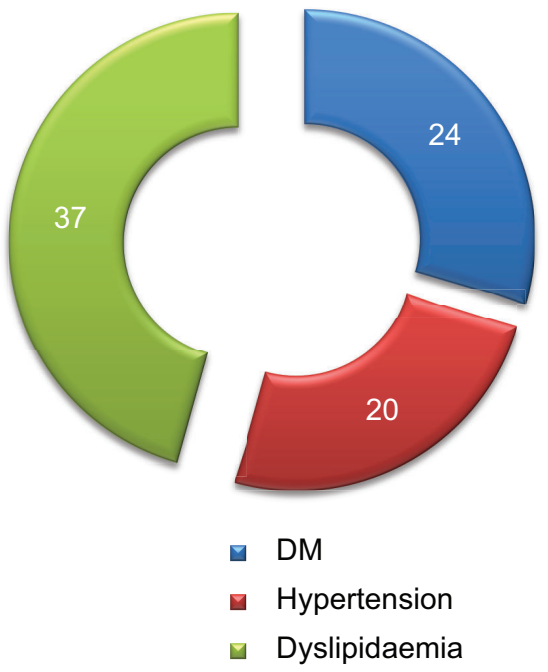

Figure 3.4: NAFLD with other co-morbid condition

Figure 3.4 showing incidence of NAFLD with DM is high, and there is correlation of NAFLD with dyslipidaemia.

\section{Associated factors with NAFLD}

The multivariate logistic regression analysis revealed that sex (adjusted $\mathrm{OR}=11.30,95 \% \mathrm{CI}=3.44-37.11, \mathrm{p}<0.0001$ ), waist circumference (adjusted $\mathrm{OR}=1.27,95 \% \mathrm{CI}=1.39-1.56$, $\mathrm{p}<0.0001$ ), waist hip ratio (Adjusted $\mathrm{C}>\mathrm{R}=0.90$, $95 \% \mathrm{CI}=0.82-0.99, \mathrm{p}=0.04$ ) and $\mathrm{BMI}$ (adjusted $\mathrm{OR}=1.83$, $95 \% \mathrm{CI}=1.10-2.20, \mathrm{p}=0.04)$ waist height ratio Adjusted $\mathrm{C}>\mathrm{R}=0.60,95 \% \mathrm{CI}=0.52-0.62, \mathrm{p}=0.04$ ) were significantly associated with NAFLD.

Table 3.8

Associated factors with NAFLD

\begin{tabular}{llccc}
\hline Factors & Beta coefficient & S.E. & Adjusted OR (95\%CI) & p value \\
\hline Sex & & & & \\
Male & 2.423 & 0.606 & $11.30(3.44-37.12)$ & $<0.0001^{*}$ \\
Female & 2.415 & 0.605 & $11.20(3.45-36.13)$ & $<0.0001^{*}$ \\
Waist circumference & 0.235 & 0.046 & $1.27(1.39-1.56)$ & $<0.0001^{*}$ \\
waist hip ratio(WHR) & 0.102 & 0.050 & $0.90(0.82-0.99)$ & $0.04^{*}$ \\
WHtR & 0.185 & 0.107 & $0.50(0.05-1.02)$ & 0.08 \\
BMI & 0.606 & 0.711 & $1.83(1.10-2.20)$ & $0.001^{*}$ \\
Constant & 31.619 & & & \\
\hline
\end{tabular}

*Significant, SE: Standard error, OR: Odd

$\mathrm{s}$ ratio, $\mathrm{CI}$ : Confidence interval 


\section{Discussion}

In the study 240 patients was undertaken to see the association of anthropometric measurement with USG diagnosed Non Alcoholic Fatty Liver Disease in Dhaka Medical College Hospital from August/September, 2013 to February/March, 2014. 240 non consecutive patients were enrolled in the study through non probability sampling technique.

Among them $51(21.25 \%)$ are USG diagnosed case of NAFLD and 189 are Non NAFLD. And out of 51 NAFLD cases $28(54.90 \%)$ are male, and $23(45.10 \%)$ are female.

The overall prevalence of NAFLD is 15 to $40 \%$ in western countries while $9-40 \%$ in Asian countries ${ }^{4}$.In two large population-based cohort studies (the Dionysos Study from Italy and the Dallas Heart Study from the USA) the prevalence of NAFLD ranged between $25 \%$ and $30 \% 10,11$ A USG based study conducted in India had showed a prevalence of 24.5 percent $^{12}$.

In the present study, the proportion of NAFLD was found to be 21.25 percent. This is in accordance with the study carried out by Wang etal. (2007) in China and Mohan et al. (2009) in Chennai, India who showed almost similar observation i.e. 24.5 percent and 32.0 percent respectively ${ }^{13,14}$.

It was also observed in the present study that male subjects constituted higher proportion $(55.0 \%)$ than the females (45\%). Similarly, Zhou et al. (2009) in China and Amarapurkar et al. (2007) in Mumbai, India also showed that the prevalence of NAFLD was higher in males as compared to females $(22.4 \%$ vs. $7.1 \%$ and $24.6 \%$ vs. $13.6 \%$ respectively) $)^{15,16}$.

In the present study, subjects with NAFLD had a higher BMI (26.29 \pm 4.41$)$ than those without $(21.48 \pm 2.60)$, which is in accordance with a study conducted in coastal regions of India which showed that the persons with NAFLD had a higher BMI (mean 25.9 \pm 4.2 ) than those without (mean 22.1 \pm 3.3$)^{2}$. In another study, among residents of two railway colonies at Mumbai, India showed high BMI $(>25)$ in NAFLD subjects ${ }^{16}$. In the present study, bivariate analysis showed that weight, BMI, waist circumference, hip circumference, WHR, WHtR all were significantly associated $(p<0.0001)$ with NAFLD. However, Wan et al. (2007) reported that BMI and WHR were the predictor of NAFLD by bivariate analysis $\left(\mathrm{x}^{2}=69.35, \mathrm{p}=<0.01\right)^{13}$.

In the present study, the multivariate logistic regression analysis showed that $\mathrm{sex}$ (adjusted $\mathrm{OR}=11.30,95 \% \mathrm{Cl}=3.44$ $37.11, \mathrm{p}=<0.0001$ ), waist circumference (adjusted $\mathrm{OR}=1.27$; $95 \% \mathrm{Cl}=1.39-1.56, \mathrm{p}=<0.0001)$ and hip circumference (adjusted $\mathrm{OR}=0.90,95 \% \mathrm{CI}=0.82-0.99, \mathrm{p}=0.0001$ ) were significantly associated with NAFLD. Lee et al. (2006) showed that the weight status [Obesity v/s Non-obesity, odds ratio (or $=4.4$ )] was associated with NAFLD after adjusting for age and metabolic risk factors ${ }^{17}$.

In the present study, the degree of obesity represented by BMI, WHtR and WHR were measured and analyzed. The statistical analysis showed BMI and WHtR had advantage on the prediction of NAFLD. In the present study, WC, WHR, WHtR, and BMI were measured, and statistical analysis showed WHR had advantages in the prediction of NAFLD over WC, WHtR, and BMI. WHR is a critical anthropometric indicator for the prediction of NAFLD.As the incidence of NAFLD is very high $(72.55 \%)$ in high WHR group. Moreover, our findings also reveal BMI also has value in the prediction of NAFLD, and BMI, WHR, and WHtR are closely associated with the occurrence of NAFLD. In the present study, although logistic regression was used to adjust the confounding factors including gender, our findings had the tendency to predict NAFLD in males due to the high proportion of males. Our findings may be more useful in the prediction of NAFLD in males. WHR is simple to measure and thus can be applied as an important anthropometric indicator to screen population with high risk for NAFLD which is beneficial for the diagnosis and treatment of NAFLD.

\section{Conclusion}

The results of the present study clearly demonstrated that there was a strong relationship between NAFLD and anthropometric parameters among adults in the studied population. It is recommended that USG of the liver should be a part of the routine health examination of obese adults. The anthropometric measurements that we suggested are simple, non invasive and reliable, however continued large scale studies are necessary to determine their validity on the prediction of NAFLD.

\section{References:}

1. Azad MAK, Quayum S L, Mohammad H, Chowdhury MAJ, Mahmood TAK, Rahim MA et al. Non alcoholic fatty liver disease (NAFLD) - a disease of new era. J Medicine $2007 ; 8: 17-27$.

2. Ramesh S, Sanyal AJ. Evaluation and management of nonalcoholic steatohepatitis. Hepatology 2005; 42:Suppl S2S12.

3. Ludwig J, Viaggiano TR, McGill DB, Oh BJ. Nonalcoholic steatohepatitis: Mayo Clinic experience with a hitherto unnamed disease. Mayo ClinProc 1980; 55: 434-438.

4. Nomura $\mathrm{H}$, Kashiwagi S. Prevalence of fatty liver in a general population of Okinawa, Japan. Jpn J Med 1988; 27: 142-9. 
5. Bellentani S, Saccoccio G. Prevalence of and risk factors for hepatic steatosis in northern Italy. Ann Intern Med 2000; 132: 112-7.

6. Tominaga k, Kurata JH. Prevalence of fatty liver in Japanese children and relationship to obesity: an epidemiological ultrasonographic survey. Dig Dis Sci 1995; 40: 2002-9.

7. Ratziu V, Giral P. Liver of fibrosis in overweight patients. Gastroenterology 2000; 118: 1117-1123.

8. Rahman M, Abedin T, Amin R, Rahman MR, Faiz MA. Non Alcoholic Fatty Liver Disease- Is It Always Benign? Journal of Bangladesh College of Physicians and Surgeons2007;9:25(3)

9. Mottin C.C., Moretto M., Padoin A.V., Swarowsky A.M.Toneto M.G., Glock L, et al. The role of ultrasound in the diagnosis of hepatic steatosis in morbidly obese patients. Obes Surg 2004; 14: 635-7.

10. Bellentani S, Tiribelli C, Saccoccio G, et al. Prevalence of chronic liver disease in the general population of northern Italy: the Dionysos Study. Hepatology 1994;20:1442-49.

11. Szczepaniak LS, Nurenberg P, Leonard D, et al. Magnetic resonance spectroscopy to measure hepatic triglyceride content: Prevalence of hepatic steatosis in the general population. Am J Physiol Endocrinol Metab 2005; 288:E462-E68.
12. Deepak Amarapurkar, Prafull Kamani, Nikhil Patel, Parijat Gupte, Pravin Kumar, Subhash Agal. Prevalence of nonalcoholic fatty liver disease: Population based study. Annals of Hepatology 2007; 6(3):161-63.

13. Wang Z, Xia B, MaC, et al. Prevalence and risk factors of Non-alcohholic fatty liver disease in Shuigouohu district of Wuhan city, central China. Postgrad Med J. 2007; 83(977): 192-195.

14. Mohan V, Farooq S, Deepa M, et al. Prevalence of Nonalcoholic fatty liver disease in urban south Indians in relation to different grades of glucose intolerance and metabolic syndrome. Diabetes Res Clin Pract. 2009; 84(1): 84-91.

15. Li H, Wang YJ, Tan K, Zeng L, Liu L, Zhou et al et al. Prevalence and risk factors of fatty liver disease in Chengdu,Southwest China. Hepatobiliary Pancreat Dis Int. 2009;8(4):377-82.

16. Amarapurkar D, Kamani P, Patel N, Gupte P, Kumar P, Agal $S$ et al Prevalence of non-alcoholic fatty liver disease: population based study. Ann Hepatol. 2007;6(3):161-26.

17. Hashimoto E, Yatsuji S, Kaneda H, Yoshioka Y, Taniai M, Tokushige $\mathrm{K}$ et al. The characteristics and natural history of Japanese patients with nonalcoholic fatty liver disease. Hepatol Res. 2005;33(2):72-6. 